\title{
Artritis reumatoide: consideraciones psicobiológicas ${ }^{*}$
}

\section{Rheumatoid Arthritis: Psychobiological Considerations}

\author{
Japcy Margarita Quiceno* \\ Universidad de San Buenaventura, \\ Bogotá, Colombia \\ Stefano Vinaccia \\ Universidad Católica de Colombia \\ Recibido: 9 de octubre de 2010 \\ Revisado: 20 de noviembre de 2010 \\ Aceptado: 2 de enero de 2011
}

\section{Resumen}

En la literatura es bien conocido el peso de las emociones negativas en la fisiopatología de la artritis reumatoide, pero hay poca información empírica del impacto de variables psicosociales positivas sobre esta enfermedad. Por tanto, el propósito de este trabajo es hacer énfasis en los factores psicobiológicos relacionados con la artritis reumatoide. Se describen las consideraciones epidemiológicas, los síntomas físicos constitutivos, los factores psicosociales más relevantes, como el papel que juegan las emociones positivas y negativas, el apoyo social, la resiliencia, la autotrascendencia y la percepción de enfermedad en la evolución y pronóstico de esta patología reumática. Conclusión: variables psicosociales positivas pueden promover la salud mental y física y aminorar el afecto negativo en pacientes con artritis reumatoide.

Palabras clave: artritis reumatoide, factores fisiológicos, factores psicosociales

\section{Abstract}

In literature the weight of negative emotions in the pathophysiology of rheumatoid arthritis is well known

* Artículo de revisión. Grupo de Investigación Salud Comportamental.

** Correspondencia: Japcy Margarita Quiceno, Universidad de San Buenaventura, Medellín, Colombia; Stefano Vinaccia, Universidad Católica de Colombia, Correo electrónico: japcyps@hotmail.com; vinalpi47@hotmail.com. Dirección postal: Avenida Caracas No. 46-40, Universidad Católica de Colombia. 
but there is little empirical evidence of positive impact of psychosocial variables on this disease. It is therefore the purpose of this paper to emphasize the psychobiological factors related to rheumatoid arthritis. We describe the epidemiological considerations, establishing physical symptoms, the most important psychosocial factors role of positive and negative emotions, social support, resilience, self-transcendence and the perception of disease in the evolution and prognosis of the rheumatic disease. Conclusion: positive psychosocial variables can promote mental and physical health and reduce negative effect in patients with rheumatoid arthritis.

Key words: rheumatoid arthritis, physiological factors, psychosocial factors

\section{Introducción}

La artritis reumatoide (AR) es una enfermedad articular, autoinmune, multisistémica, inflamatoria y crónica, cuyo órgano blanco principal es la membrana sinoviál. Afecta principalmente a las mujeres en una proporción 3 a 1 en el mundo con relación a los hombres y su prevalencia en la población latinoamericana es cercana al 0,5\% (Delgado-Vega, Martín, Granados \& Anaya, 2006; Gutiérrez \& Londoño, 2004). La prevalencia aumenta con la edad y las diferencias entre género disminuyen en el grupo de población de edad avanzada. El inicio de la enfermedad es más frecuente durante el cuarto y quinto decenios de la vida y el pico de incidencia ocurre entre los 35 y 50 años de edad -80\% de los casos- (Ramos, 1999).

Aunque su causa es desconocida, se han identificado factores endocrinos, ambientales y genéticos involucrados en su desarrollo, los cuales pueden variar de una población a otra. La existencia de agregación familiar indica su carácter hereditario. Sin embargo, la herencia de la AR es poligénica y no sigue un patrón mendeliano (Delgado-Vega et ál., 2006; Gutiérrez \& Londoño, 2004). Entre los factores relacionados se encuentra el posible efecto modulador de anticonceptivos orales, la fertilidad, la terapia de reemplazo estrogénico, la edad de la menarquia, la fertilidad y el embarazo. Otros factores relacionados y que tienen un efecto modulador sobre el pronóstico de la enfermedad son la educación, el estatus marital, el nivel socioeconómico y el inicio de la terapia luego del comienzo de los síntomas, la adicción al tabaco y la dieta. Con frecuencia la AR compromete otros órganos distintos a las articulaciones y tiene un impacto adverso en la esfera biopsicoso- cial (Gutiérrez \& Londoño, 2004; Rojas-Villarraga et ál., 2009).

La incapacidad física asociada con la AR afecta el campo laboral del paciente dificultándole mantener un empleo estable. La incapacidad laboral entre los 5 y 10 años desde el inicio de la enfermedad en diferentes países es del $43 \%$ al $50 \%$, en muchos casos no vuelven a trabajar o cuando lo hacen se encuentran con que deben ocupar un cargo de menor responsabilidad (Cadena et ál., 2003). Los costos económicos directos, indirectos e intangibles de la AR son enormes, en Colombia es la segunda causa de perdida de años saludables por discapacidad en mujeres entre los 15 y los 44 años de edad (Caballero, 2004). No obstante, no se dispone en Colombia de información fidedigna sobre la prevalencia e incidencia (Gutiérrez \& Londoño, 2004). Aunque la AR ocurre en todas las razas, países y culturas del mundo, en estudios colombianos se ha encontrado que ésta es menos severa en pacientes de raza negra. Esto se debe en parte a que los pacientes no tienen los genes de susceptibilidad que tienen las personas de raza blanca y mestiza. Por el contrario, los pacientes con genes susceptibles la desarrollan más severamente, así como aquellos que tienen los anticuerpos anticitrulina la desarrollan a una edad menor que aquellos que no los tienen (Anaya, González \& Rojas-Villarraga, 2009). En estudios realizados por Rojas-Villarraga et ál. (2009) en Colombia, se ha encontrado que el curso de la AR y su pronóstico varía de un paciente a otro, ya que los resultados han indicado que el antecedente familiar en primer grado de AR, la presencia en suero de anticuerpos anti-CCP y un marcador genético (HLA-DRB1*0404) son los factores asociados con el deterioro articular en pacientes colombianos. 


\section{Fisiopatología de la artritis reumatoide}

Las células del sistema inmune tales como linfocitos y monocitos entran en el espacio articular y liberan sustancias químicas que producen inflamación, tales como: enzimas, citoquinas, óxido nítrico, prostaglandinas que causan dolor y daño a las diferentes partes de las articulaciones como el cartílago y el hueso o inflamación persistente de la membrana sinovial que afecta habitualmente las articulaciones periféricas -metacarpofalángicas, metatarsofalángicas, rodillas y codos generalmente- (Anaya, 1999). Dado que es el propio sistema inmune del paciente el que ataca las articulaciones y que no se ha identificado un agente externo que cause la enfermedad, la AR es considerada entonces una enfermedad autoinmune (Anaya et ál., 2009).

Los síntomas de inicio son fundamentalmente inflamación de una o más articulaciones ("actividad de la enfermedad") que consiste en el aumento del volumen de la articulación (hinchazón) con disminución del movimiento y el aumento del calor local. Además, se puede presentar rigidez matinal, el paciente siente entumecimiento e incapacidad para mover una o varias articulaciones por más de quince minutos al despertarse, y por último, se pueden presentar síntomas constitucionales como sensación de fatiga, falta de energía y fuerza, fiebre, cansancio y pérdida de peso sin motivos aparentes (Anaya et ál., 2009; Ballina \& Rodríguez, 2000). Con respecto a la fatiga, algunos estudios han encontrado relaciones significativas entre esta variable con la discapacidad, con mayor dolor, con frecuentes trastornos del sueño, con mayores niveles de depresión y con bajos niveles de autoeficacia (Pollard, Choy, Gonzalez, Khoshaba \& Scott, 2006; Wolfe \& Michaud, 2009). Por esta línea Zautra, Fasman, Parish y Davis (2007) encontraron en un estudio con pacientes de osteoartritis, artritis reumatoide y fibromialgia que la fatiga se correlacionó con un menor efecto positivo y el dolor diario se asoció con un aumento de la fatiga. Concluyendo en este estudio que la fatiga es una característica común de las enfermedades reumáticas. La fatiga ade- más se encuentra presente en los pacientes con enfermedades reumáticas entre un $50 \%$ y un $80 \%$ (Pelechano, 2008).

En cuanto a la evolución de la AR puede ser muy variable, desde sólo un proceso oligoarticular (pocas articulaciones) de breve duración y con lesiones articulares mínimas hasta una poliartritis (múltiples articulaciones) progresiva que evolucionan hasta deformar de manera importante las articulaciones. La mayoría de los pacientes presentan una evolución intermedia entre estos dos extremos. La progresión de la enfermedad va produciendo una degeneración de los cartílagos y un debilitamiento en los tendones, lo cual produce fuertes dolores y una reducción progresiva de la movilidad (Anaya, 1999). La incapacidad creada por la artritis se incrementa por lo general en los primeros años del diagnóstico, para posteriormente volverse más lenta al final de la vida del paciente; es posible que después de treinta años de persistencia se acelere otra vez con todos sus síntomas (Leight, Frics \& Parkh, 1992). La AR reduce la vida de las personas que la padecen entre 3 y 13 años frente a las que no la sufren (Ramos, 1999).

En la AR se pueden presentar además manifestaciones extraarticulares como: 1 . la anemia (hemoglobina $>12$ gramos \%) debido a la inflamación crónica, la falta de hierro y posibles hemorragias digestivas; 2 . los nódulos que aparecen en áreas de extensión de las articulaciones, particularmente en codos y en las manos; 3. problemas oculares tipo ojo seco, o síndrome de sjögren secundario; 4. vasculitis, puede manifestarse en la piel con brotes, úlceras y necrosis digital (disminución de la sangre en los tejidos de los dedos o incluso gangrena); 5 . serositis, inflamación de las membranas que recubren el pulmón (pleura) y corazón (pericardio) causando dolor, dificultades para respirar, fiebre y tos -en algunos casos no se presenta manifestación clínica-; y 6. osteoporosis con la pérdida de calcio de los huesos que los vuelve frágiles aumentando la posibilidad de fracturarse aún en pequeños accidentes (Anaya et ál., 2009).

Por tanto, todo el esfuerzo médico se centra en lograr la "remisión de la enfermedad", es decir, 
en paliar o minimizar el deterioro y alcanzar la disminución de la inflamación por medio de diferentes fármacos (Ballina \& Rodríguez, 2000). El tratamiento farmacológico se divide en sistémico que consiste en tomar o inyectarse medicamentos que van a actuar a diferentes niveles del organismo, disminuyendo de esa forma el compromiso de las articulaciones, y el local que consiste en medicamentos que se aplican a nivel de las articulaciones bursas $u$ otras zonas para disminuir la inflamación local (Anaya \& Rojas-Villarraga, 2009).

Los diferentes fármacos en dosis altas y durante tiempo prolongado pueden producir diversos efectos secundarios (Ballina \& Rodríguez, 2000). Los antiinflamatorios (AINES), como por ejemplo el ibuprofeno, voltaren, ácido acetil salicílico pueden producir toxicidad a nivel del tracto gastrointestinal, renal y cardiovascular. Los corticoides (esteroides) incrementan las infecciones, las afecciones en la piel producen efectos sobre el metabolismo, incrementa el colesterol y la glicemia y favorece la osteoporosis. Los medicamentos antirreumáticos modificadores del curso de la enfermedad (DME), como por ejemplo el metotrexate, leflunomida y los antimaláricos (hidroxicloroquina y cloroquina) pueden causar problemas digestivos, hepáticos, cutáneos y oculares. Y por último, los medicamentos biológicos anti TNF (Tumor Necrosis Factor) como por ejemplo el etanercept, infliximab y el rituximab pueden generar riesgo de infecciones, alteraciones cutáneas, daños en el sistema inmune y riesgo de infecciones (Anaya \& Rojas-Villarraga, 2009). En caso crítico como de dolor extremo o recrudecimiento puede ser necesario la intervención quirúrgica (Taylor, 2007).

\section{Factores psicosociales asociados a la artritis reumatoide}

La AR afecta al individuo a nivel cognitivo, emocional y comportamental desde el momento en que recibe el diagnóstico. El paciente se ve enfrentado a varios procesos interactuantes desde los factores fisiológicos asociados que llevan a la pérdida de un gran número de funciones de la vida diaria, que afectan en mayor o menor grado el desplazamiento, el cuidado personal, el tra- bajo y otras actividades básicas, alterando directa o indirectamente la independencia económica y los roles sociales de la persona, hasta los factores psicoemocionales que también se ven implicados (González, 2004; Isik, Koca, Ozturk \& Mermi, 2007).

\section{Emociones negativas}

Las personas con AR experimentan una serie de emociones negativas ante la discapacidad creada por la enfermedad (Cadena, Cadavid, Ocampo, Vélez \& Anaya, 2002; Smith \& Zautra, 2002). En diferentes estudios se ha encontrado como principales factores psicológicos la depresión/desesperanza, la ansiedad (Azad, Gondal \& Abbas, 2009; Hommel, Wagner, Chaney, White \& Mullins, 2004) el estrés (Straub \& Kalden, 2009) y los trastornos del sueño (Buenaver \& Smith, 2007).

Al respecto, en un estudio donde se pretendió categorizar las causas del $59 \%$ de la discapacidad en pacientes con AR basándose en el modelo según el cual la patología conduce al impedimento, a la incapacidad funcional y finalmente a la discapacidad, se encontró que la discapacidad en estos pacientes podía atribuirse en un $26 \%$ a factores psicológicos, a síntomas depresivos y a otros modificadores externos, mientras que el $33 \%$ podía atribuirse directamente a la enfermedad (Escalante \& Del Rincón, 1999).

La prevalencia de la depresión en la AR es superior a las tasas de depresión de la población en general $(2-4 \%)$ y en atención primaria $(5-10 \%)$ y sobre la base de la evaluación psiquiátrica y diagnóstico clínico de depresión la prevalencia oscila entre el $13 \%$ al $20 \%$, y es considerablemente más elevada cuando se emplean instrumentos psicométricos de auto informe. La depresión se asocia estrechamente con dolor o mayor número de articulaciones dolorosas, duración de la enfermedad, incapacidad laboral y funcional -que representa ausentismo laboral y consecuente pérdida económica y social-, utilización de los servicios de salud, pobre adherencia al tratamiento, suicidio, distorsiones cognitivas, ser joven, velocidad de sedimentación globular (VSG) elevada, ausencia de pareja, estrés diario, ansiedad, desesperanza aprendida, baja autoeficacia (Bazzichi et ál., 2005; 
Caballero-Uribe et ál., 2004) y calidad de vida (Cadena et ál., 2003; Hill et ál., 2007). Por otro lado, se ha encontrado que la depresión, la ansiedad y el dolor, más que ser una consecuencia son síntomas constitutivos de la etiopatología de la AR debido al efecto directo de las citoquinas sobre el sistema nervioso central (Bagnato et ál., 2006).

En cuanto al estrés causado por la AR depende del tiempo de evolución de la enfermedad y puede darse al inicio o en una recaída. Es posible que en un estadío temprano y rápidamente progresivo la percepción de estrés sea mayor, mientras que si la enfermedad se ha establecido a lo largo de los años el paciente puede aprender a vivir con la enfermedad, $\mathrm{y}$, por tanto, el grado de estrés psicológico puede ser menor (González, 2004). En pacientes con AR en comparación con grupos control sanos, la respuesta inmunológica es más fuerte frente al estrés, afectándose las funciones homeostáticas, esto puede explicar en parte el deterioro de la enfermedad (Taylor, 2007). De otro lado, la percepción de estrés es mucho mayor en estos pacientes en comparación con población en general (González, 2004).

El estrés en los pacientes con enfermedades inflamatorias crónicas como la AR es común y estimula mecanismos proinflamatorio afectando la salud física en general. La activación de los sistemas de respuesta del estrés influye en la estrecha relación del sistema nervioso simpático, el eje hipotalámico-hipofisario-adrenal (Eje HHA) y el sistema inmunológico. Entre otros mecanismos, la pérdida de las fibras nerviosas simpáticas en el tejido inflamado y la insuficiencia de la secreción de cortisol en relación con la inflamación llevan a una mayor carga proinflamatoria en la AR. El estrés y la consiguiente estimulación de la inflamación (sistémica y local) pueden conducir a una mayor sensibilización del dolor y aún más, a los efectos de los sistemas de respuesta del estrés, es decir, el círculo vicioso del estrés, el dolor y la inflamación (Cutolo \& Straub, 2006; Geenen, van Middendorp, Bijlsma, 2006; Straub \& Cutolo, 2001; Straub \& Kalden, 2009).

Respecto a los trastornos del sueño se ha encontrado que aqueja a un $50 \%$ de las personas con enfermedades reumáticas (Buenaver \& Smith, 2007). En pacientes con AR su prevalencia es alta especialmente en personas mayores de 55 años, presentándose relaciones entre las quejas sobre el sueño con la actividad de la enfermedad, los niveles de depresión, la privación del sueño y las citoquinas (Abad, Sarinas \& Guilleminault, 2008). Algunos autores argumentan que el estrés es una variable que interfiere significativamente en la privación del sueño (Treharne et ál., 2007). Mientras que otros autores plantean que es el dolor el que interfiere en el sueño, y que a su vez el mismo trastorno del sueño exacerba el dolor. Al respecto datos epidemiológicos indican que hasta un $60 \%$ de las personas con AR experimentan dolor durante la noche (Davis, 2003). Además, se ha encontrado que la fragmentación del sueño se asocia con aumento de dolor y en las horas de la mañana con rigidez en los adultos (Buenaver \& Smith, 2007).

\section{Emociones positivas}

Fredrickson (1998, 2001) propuso el modelo de "ensanchar y construir" emociones positivas ("broaden-and-build theory of positive emotions"). Según este modelo hay tres efectos secuenciales de las emociones positivas: 1. Ampliación: las emociones positivas pueden ampliar tanto el pensamiento como la acción; 2. Construcción: debido a la ampliación, se favorece la construcción de recursos personales para afrontar situaciones difíciles, y 3. Transformación: la construcción origina la transformación de la persona, haciéndola más creativa, con un conocimiento más profundo de las situaciones, haciéndola más resistente ante las dificultades de la vida y posibilitándole una mejor integración social, esto en ultima instancia propiciaría y generaría la experimentación de nuevas emociones positivas ("espiral ascendente").

La evidencia empírica sugiere, además, que existe una relación marcada positiva entre los estados psicológicos y la salud. A nivel biológico se han encontrado asociaciones de las emociones positivas con un aumento de la resistencia a la infección, menores niveles de cortisol y disminución de los marcadores inflamatorios como la interleu- 
cina-6 (Steptoe, Dockray \& Wardle, 2009) y reducción en los niveles de dolor (Fredrickson \& Losada, 2005). A nivel psicosocial las emociones positivas se asocian con la protección psicosocial (mayor conexión social y apoyo social percibido), el optimismo, un estilo de afrontamiento más adaptativo, aumenta la felicidad, favorece en la madurez psicológica y la longevidad (Fredrickson \& Losada, 2005), los sentimientos de gratitud (Fredrickson, 2004), la resiliencia cuando se padece una enfermedad crónica (Philippe, Lecours \& Beaulieu-Pelletier, 2009) y la religión y la espiritualidad. Ya que las emociones positivas que genera la espiritualidad fomentan la sensación de que la vida tiene sentido, ayudan a encontrar un significado positivo a los acontecimientos cotidianos y a los momentos de adversidad, y promueven el sentido de coherencia (Saroglou, Buxant \& Tilquin, 2008). Se ha encontrado además que las creencias y los estados positivos mejoran la función inmune, favorece en la toma de decisiones médicas, pueden desempeñar un papel importante a largo plazo en el control de riesgos sobre la salud y de la aparición de enfermedades crónicas, sugiriendo la importancia de las intervenciones en esta área ya que se promueve el bienestar psicológico y la salud en lo que se refiere a la progresión de la enfermedad (Aspinwall \& Tedeschi, 2010).

Específicamente en estudios con pacientes de AR se ha encontrado que el percibir y experimentar emociones intensamente, predice una disminución del afecto positivo, y la dificultad para reconocer y expresar las emociones pronostica un aumento de la actividad de la enfermedad (van Middendorp, Geenen, Sorbi, van Doornen \& Bijlsma, 2005). En otro estudio con pacientes de AR se encontró que el dolor aunque es parcialmente influido por el estado de la enfermedad y las variables demográficas es más afectado por los estados emocionales positivos y negativos, por tanto, se plantea la importancia de trabajar a nivel de intervención día a día sobre la regulación emocional positiva y negativa en estos pacientes, ya que es clave para entender la experiencia de dolor (Connelly et ál., 2007).

\section{Apoyo social}

El apoyo social hace referencia al "contexto funcional de las relaciones, integrando el grado en que las relaciones sociales implican elementos afectivos o emocionales, instrumentales o de ayuda tangible, información, etc." (Rodríguez-Marín \& Neipp, 2008, p. 102).

A nivel general la literatura plantea que el apoyo social reduce la tendencia a padecer enfermedades, favorece la recuperación cuando se tiene alguna enfermedad, reduce el riesgo de mortalidad en enfermedades crónicas, favorece la adherencia a los tratamientos médicos y favorece en los procesos cognitivos a medida que se avanza en edad (Gómez, Pérez \& Vila, 2001).

En lo que respecta a estudios con muestras de pacientes con diagnóstico de AR, se ha encontrado que el apoyo social tiene un peso significativo en el bienestar de estos pacientes en general (Krol, Sanderman \& Suurmeijer, 1993). En Irlanda Minnock, FitzGerald y Bresnihan (2003) con una muestra de 58 pacientes de AR y sus cuidadores primarios encontraron que éstos manifestaron tener niveles más altos de satisfacción con el apoyo social de familiares y amigos y, hubo además, asociaciones positivas entre el apoyo social con diferentes dimensiones físicas, emocionales y de bienestar social del cuestionario de impacto de la artritis reumatoide (AIMS). Además, Strating, Suurmeijer y van Schuur (2006) en Holanda con una muestra de 129 pacientes de AR desarrollaron un estudio longitudinal encontrándose que a corto plazo los factores relacionados con la enfermedad (dolor articular, dolor e incapacidad funcional) y el apoyo social eran importantes en la determinación del estrés psicológico. Además, se encontró un efecto amortiguador del acompañamiento. Mientras que a largo plazo los factores relacionados con la enfermedad siguieron siendo importantes en la determinación del estrés psicológico, pero en menor medida. En conclusión, se planteó en este estudio, que es más determinante a corto plazo que a largo plazo el apoyo social en los pacientes con AR. Por otro lado, Zyrianova et ál. (2006) en Irlanda, con una muestra de 68 adultos con AR encontraron después de contro- 
lar el efecto de la edad, el sexo, el estado civil y la duración de la artritis, que la percepción de apoyo social es un predictor independiente, altamente significativo de la depresión y la ansiedad en estos pacientes. Los autores concluyen la importancia del apoyo social como un factor coadyuvante en los procesos de tratamiento de ansiedad-depresión en pacientes con AR.

Más concretamente en poblaciones latinas y en Colombia el apoyo familiar es de vital importancia en los pacientes con AR (Abraído-Lanza, 2004; Cadena et ál., 2003; González, 2004). En la cultura colombiana hay una tendencia de la familia y sus generaciones de involucrarse en el cuidado de los enfermos y brindar apoyo a nivel emocional como económico (Cadena et ál., 2002, 2003).

\section{Resiliencia y su relación con autoes- tima, autoeficacia y autocontrol}

La autoestima, la autoeficacia y la autorregulación o el autocontrol o la percepción de control y el lugar de control interno, son considerados por los hallazgos empíricos sobre resiliencia como factores protectores ante el estrés y los síntomas de la enfermedad (Juth, Smyth \& Santuzzi, 2008; Smith \& Zautra, 2008).

A nivel general, en estudios sobre resiliencia con pacientes crónicos se ha encontrado que la autoestima y la confianza en sí mismo promueven la adaptación a la enfermedad (Kralik, van Loon \& Visentin, 2006). En otras investigaciones se ha encontrado que las personas que viven con una enfermedad crónica y que se enfrentan a periodos de hospitalización desarrollan procesos de crecimiento o aprendizaje y disfrutan incluso más la vida (Vera, Carbelo \& Vecina, 2006). Se ha hallado además, que las personas resilientes aparte de tener una alta autoestima y estabilidad emocional poseen altos niveles fisiológicos de endorfinas. Los efectos positivos de la resiliencia sobre la fisiología y la salud probablemente funcionan a través del afrontamiento (Horner, 1998). Al respecto, Lundman et ál. (2010) plantean que poseer altos niveles de resiliencia posibilita regular los efectos negativos del estrés, a su vez que se aumenta la autoestima, el control personal y las expectativas de autoeficacia que en momentos de enfermedad favorecen la sobrevivencia y la búsqueda de un afrontamiento más adaptativo.

Algunos estudios indican que reforzar tanto la autoestima como el lugar de control interno ha sido asociado con bajos niveles de reactividad de cortisol en situaciones de estrés (Simeon et ál., 2007). Por consiguiente, estudios de resiliencia en muestras de pacientes con AR en los que se ha evaluado el dolor corporal, han hallado que los efectos positivos de la resiliencia en relación con el dolor resultan del incremento del bienestar personal, y también de los cambios en la evaluación cognitiva de autoeficacia que promueven otras nuevas formas de afrontamientos al dolor (Strand et ál., 2006). En un estudio sobre resiliencia con pacientes con osteoartritis Wright, Zautra y Going (2008) plantearon que los efectos de la resiliencia sobre el dolor fueron mediados a través de la autoeficacia, indicando que una alta autoeficacia estaba relacionada con bajo dolor y mejor funcionamiento físico, es decir, la autoeficacia funcionaría como un antídoto contra el dolor. En otros estudios con este mismo tipo de enfermedad se ha encontrado que factores como las emociones positivas, la vitalidad, la extraversión han mostrado ser predictores que incrementan la autoeficacia, la actividad física y el afrontamiento (Wright et ál., 2008). Al respecto, Maddux y Gosselin (2003) han enfatizado que una de las consecuencias más importantes del desarrollo de creencias de autoeficacia es el desarrollo de la capacidad para la autorregulación. Los datos empíricos son concluyentes en los beneficios para la salud de la resiliencia como variable protectora y amortiguadora ante situaciones que implican altos niveles de amenaza.

\section{Autotrascendencia}

A finales de los años ochenta del siglo XX la enfermera Pamela $G$. Reed desarrolla la teoría de la autotrascendencia como un recurso psicosocial y espiritual importante en el desarrollo madurativo de las personas, como cualquier otra capacidad evolutiva del individuo necesaria para conseguir 
sensación continua de plenitud y de conexión consigo mismo y el entorno. La autotrascendencia se le considera además como proceso dinámico que puede ser una poderosa estrategia de afrontamiento en tiempos de adversidad y vulnerabilidad, es decir, cuando la persona se encuentra ante experiencias de estrés físico, emocional y espiritual (Neill, 2005; Teixeira, 2008).

Por otro lado, la autotrascendencia es definida como una ampliación de los límites autoconceptuales de forma multidimensional: (a) hacia dentro a través de experiencias introspectivas; (b) hacia afuera aumentando las relaciones con los demás; (c) temporalmente, mediante la integración del pasado y el futuro en el presente (Reed, 1991); y (d) mediante la conexión con dimensiones que están más allá del mundo perceptible -un poder más alto- (Coward, 2007; Reed, 2003). Además, Pamela G. Reed plantea que: "dado que la autotrascendencia es de naturaleza multidimensional, podrían añadirse otras dimensiones con el fin de describir la capacidad de ampliar los límites autoconceptuales" (Coward, 2007, p. 649). Por lo anterior, la autotrascendencia y la espiritualidad pueden superponerse (Teixeira, 2008).

Los tres conceptos teóricos del modelo de Reed (2003) son la vulnerabilidad, la autotrascendencia y el bienestar. La vulnerabilidad hace referencia a la conciencia de la persona de ser un ser mortal, ésta se ve aumentada en la etapa del envejecimiento $\mathrm{u}$ otras etapas del ciclo vital cuando aparece una enfermedad, un traumatismo o una crisis vital. La autotrascendencia se refiere a las fluctuaciones que tienen lugar en la percepción de los límites que hacen que las personas vayan más allá de sus puntos de vista inmediatos y restringidos sobre sí mismos y el mundo. Y el bienestar significa sensación de plenitud y salud, de acuerdo con los criterios que cada quien tiene de lo que considera plenitud y salud. En estos tres conceptos se ven implicados factores moderadores-mediadores y los puntos de intervención. Los factores moderadores-mediadores son variables personales y contextuales como la edad, el género, las experiencias vitales y el entorno social que pueden influir en la relación existente entre la vulnerabilidad y la autotrascendencia y entre la autotrascendencia y el bienestar. Los puntos de intervención son dos, que interactúan de alguna forma con el proceso de autotrascendencia: como las acciones del terapeuta que pueden centrarse directamente en un recurso interno del paciente que le capacita para la autotrascendencia o puede centrarse en algún factor personal o contextual que afecta la relación entre vulnerabilidad y autotrascendencia y entre autotrascendencia y bienestar. En síntesis, cuando aumenta la vulnerabilidad, aumenta la autotrascendencia; y la autotrascendencia está relacionada positivamente con el bienestar. En la Figura 1 se puede apreciar el modelo de Reed (2003). Reed (1987) basándose en sus planteamientos teóricos desarrolla la escala de autotrascendencia (Self-Transcendence Scale, STS).

A partir de este modelo se cuenta con un número significativo de investigaciones que plantean las relaciones positivas entre autotrascendencia, bienestar y calidad de vida en diferentes enfermedades crónicas como VIH/Sida, cáncer de mama, trasplante de hígado, enfermos terminales, cáncer de próstata y artritis reumatoide (Coward, 2007).

Al respecto, Neill (2002) encontró en historias de mujeres con AR que éstas revelaron la transcendencia de los límites personales y de transformación personal como nuevos modos de vida, que incluían los "placeres simples" y "ser positivo". Más adelante Neill (2005) encuentra en otro estudio con mujeres con diagnóstico de AR y esclerosis múltiple que los "placeres simples" y "ser positivo" además de ganar autocontrol y autodeterminación son otras formas de autotrascendencia. En este último estudio la autotrascendencia y la transformación personal serian asimilables a una forma de afrontamiento. De otro lado, Quiceno (2010) encontró altos niveles de autotrascendencia en muestras de pacientes colombianos con AR y puntos de contacto entre esta variable con la resiliencia. Finalmente, se cuenta con programas de intervención que buscan promover los pensamientos y actividades relacionadas con la autotrascendencia en las personas que tienen la necesidad de aumentar su sentido de plenitud y bienestar (Coward, 2003). 


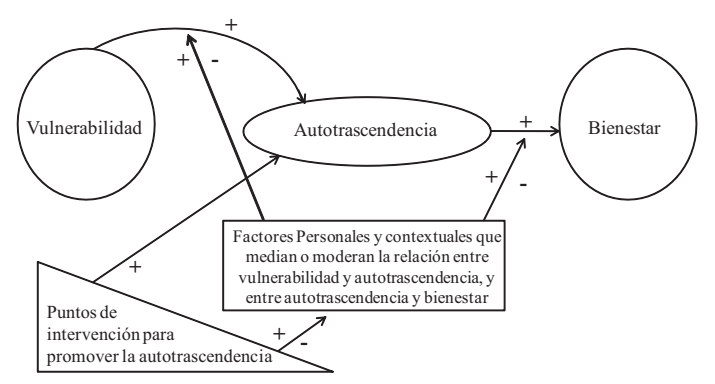

Figura 1. Modelo de la teoría de la autotrascendencia (Reed, 2003).

\section{Percepción de enfermedad}

En principios de los años ochenta (s. XX) el psicólogo Howard Leventhal desarrolla el modelo de sistemas autorregulatorios. El supuesto básico de este modelo es la consideración del paciente como un procesador activo que construye o elabora una definición o representación de su enfermedad y que en función de esta representación regula su comportamiento. A partir de su modelo Howard Leventhal plantea además que las representaciones/esquemas cognitivos que los pacientes elaboran sobre sus enfermedades incluyen diferentes factores como: 1. Identidad, que hace referencia a los signos, síntomas y la etiqueta de la enfermedad; 2 . Consecuencias, son las consecuencias físicas, sociales y económicas percibidas de la enfermedad, y las consecuencias emocionales sentidas; 3 . Causas, etiología o las causas percibidas de la enfermedad; 4. Duración (cronograma), marco temporal percibido para el desarrollo y la duración de la amenaza de enfermedad: y 5. Cura/control, el punto hasta que la enfermedad es receptiva al tratamiento (Quiceno \& Vinaccia, 2010).

A partir de este modelo se diseñó el cuestionario de percepción de enfermedad (Illness Perception Questionnaire, IPQ) dándose en los últimos cinco años un aumento progresivo de investigaciones en el área, con el objetivo de explorar la naturaleza de varias enfermedades y la relación entre las percepciones de la enfermedad y la adaptación (Quiceno \& Vinaccia, 2010).
En cuanto a las enfermedades reumáticas y más específicamente la AR se cuenta con algunos estudios desarrollados con las diferentes versiones del cuestionario de percepción de enfermedad IPQ encontrándose que las consecuencias, la identidad, el control personal y la respuesta emocional son las dimensiones que han tenido implicaciones para la adaptación a la enfermedad y la calidad de vida física y mental (Quiceno \& Vinaccia, 2010).

Al respecto, Scharloo et ál. (1998) en un estudio en Holanda encontraron en 244 pacientes con diagnóstico artritis reumatoide, enfermedad pulmonar obstructiva crónica y psoriasis medidos con el IPQ, que un afrontamiento pasivo, una fuerte identidad, la creencia de una larga duración de la enfermedad, la creencia de consecuencias graves y desfavorables resultados médicos se asociaron con una peor función general y social del cuestionario MOS SF-20. Mientras que el afrontamiento en la búsqueda de apoyo social y las creencias de cura/control de la enfermedad fueron significativamente relacionadas con un mejor funcionamiento general. Además Scharloo et ál. (1999) en Holanda y Gran Bretaña encontraron en 71 pacientes con AR que la creencia en consecuencias negativas de la enfermedad se asoció con mayores visitas médicas y puntuaciones más altas en ansiedad y cansancio. Un control menor y menos percepción de la expresión emocional se asociaron con más ingresos hospitalarios. La creencia de que la enfermedad dura por largo tiempo se asoció con altos niveles de ansiedad.

Por otro lado, Murphy, Dickens, Creed y Bernstein (1999) en Inglaterra encontraron en 62 pacientes de AR evaluados con el IPQ asociaciones entre síntomas depresivos -aún controlando la discapacidad generada por la enfermedad- y una percepción de consecuencias más negativas de la enfermedad y el control de ésta. Por otro lado, hubo uso de estrategias de afrontamiento negativas. Este estudio indicó la estrecha relación entre la depresión y una percepción negativa de la enfermedad.

Sharpe, Sensky y Allard (2001) en Australia y Gran Bretaña encontraron en 22 pacientes de AR que 
un conjunto de cinco factores predecían la depresión: el nivel inicial de la depresión, la discapacidad, el dolor, las creencias acerca de las consecuencias de la artritis y las estrategias de afrontamiento.

Groarke, Curtis, Coughlan y Gsel (2004) en Irlanda encontraron en 75 pacientes de AR que varios aspectos de ajuste como una buena función física, bajo dolor y baja depresión (medido con el cuestionario de impacto de la artritis -AIMS-) se asociaron con baja identidad, alta cura/control, consecuencias más graves y larga duración de la enfermedad (medido con el IPQ). Este estudio concluye que la percepción de la enfermedad tiene importantes implicaciones para la adaptación a la enfermedad y compensa los efectos del estado de la enfermedad sobre la depresión, la función física y el dolor.

Bijsterbosch et ál. (2009) en Holanda encontraron en pacientes con osteoartritis que la percepción de la enfermedad cambiaba con el tiempo y que estos cambios estaban relacionados con la progresión de la discapacidad, lo que sugiere la posible intervención en el área. Los pacientes que les progresaba la discapacidad tuvieron un aumento en los síntomas de la enfermedad, percibían mayores consecuencias de ésta, tenían una percepción de cronicidad, de mayor respuesta emocional y menor control personal y de una pobre comprensión de la enfermedad. Además, el tener niveles basales altos a nivel de los síntomas estaba asociado con niveles altos de consecuencias y con niveles bajos de control personal sobre la enfermedad, siendo esto un factor predictivo de discapacidad alta al cabo de seis años.

\section{Conclusión}

La literatura ha hecho énfasis y hay un sinnúmero de información sobre el impacto de las emociones negativas (depresión/desesperanza, ansiedad, estrés) en la fisiopatología de la AR y de éstas sobre la calidad de vida, mientras que hay poca información empírica sobre el papel de las variables psicosociales positivas como las emociones positivas, el apoyo social, la resiliencia y variables es- pirituales como la autotrascendencia que pueden servir de marcos protectores para un mejor bienestar psicológico y físico y mitigar las emociones negativas. Lo mismo puede decirse acerca de la percepción de enfermedad que es una variable moduladora que puede influir positiva o negativamente sobre los mismos factores psicosociales y fisiológicos de la AR. Esto implicaría la relevancia de programas de intervención focalizados desde la psicología positiva para abordar de forma integral el impedimento, la discapacidad y minusvalía-desventaja social que acarrea esta patología.

Por tanto, los factores psicosociales tienen un peso importante sobre la artritis reumatoide, pero discernir específicamente cual de éstos es más relevante sobre la evolución y pronóstico de la AR es complejo, ya que se pueden entrelazar o solapar entre sí o presentar comorbilidad, porque están asociados a factores de fisiopatología de la AR más o menos agresivos que llevan a que el peso de la salud mental se torne más distal o proximal a través del tiempo.

\section{Referencias}

Abad, V.C., Sarinas, P.S. \& Guilleminault, C. (2008). Sleep and Rheumatologic Disorders. Sleep Medicine Reviews, 12(3), 211-128.

Abraído-Lanza, A.F. (2004). Social Support and Psychological Adjustment Among Latinas with Arthritis: a Test of a Theoretical Model. Annals of Behavioral Medicine, 27(3), 162-171.

Anaya, J.M. (1999). Genes y Artritis. Revista Colombiana de Reumatología, 6, 240-250.

Anaya, J.M., González, H. \& Rojas-Villarraga, A. (2009). Respuestas a las principales preguntas. En J.M. Anaya y A. Rojas-Villarraga (Eds.). Manual para pacientes con artritis (pp. 5-18). Bogotá, Colombia: CIB.

Anaya, J.M. \& Rojas-Villarraga, A. (2009). Tratamiento de la artritis reumatoide. En J.M. Anaya \& A. Rojas-Villarraga. (Eds.). Manual para pacientes con artritis (pp. 33-50). Bogotá, Colombia: CIB. 
Aspinwall, L.G. \& Tedeschi, R.G. (2010). The Value of Positive Psychology for Health Psychology: Progress and Pitfalls in Examining the Relation of Positive Phenomena to Health. Annals of Behavioral Medicine, 39(1), 4-15.

Azad, N., Gondal, M. \& Abbas, N. (2009). Frequency of Depression and Anxiety in Patients Attending a Rheumatology Clinic. Journal of the College of Physicians and Surgeons-Pakistan, 18(9), 569-573.

Bagnato, G., De Filippis, L.G., Caliri, A., Bruno, A., Gambardella, N., Muscatello, M.R. et ál. (2006). Comparation of Levels of Anxiety and Depression in Patients with Autoimmune and Chronic-degenerative Rheumatic: Preliminary Data. Reumatismo, 8(3), 206-211.

Ballina, F. \& Rodríguez, A. (2000). Artritis Reumatoide. Revista Española de Reumatología, 27, 56-64.

Bazzichi, L., Maser, J., Piccinni, A., Rucci, P., Del Debbio, A., Vivarelli, L. et ál. (2005). Quality of Life in Rheumatoid Arthritis: Impact of Disability and Lifetime Depressive Spectrum Symptomatology. Clinical and experimental rheumatology 23(6), 783-788.

Bijsterbosch, J., Scharloo, M., Visser, A.W., Watt, I., Meulenbelt, I., Huizinga, T.W.J. et ál. (2009). Illness Perceptions in Patients with Osteoarthritis: Change Over Time and Association with Disability. Arthritis \& Rheumatism, 61(8), 1054-1061.

Buenaver, L.F. \& Smith, M.T. (2007). Sleep in Rheumatic Diseases and Other Painful Conditions. Current Treatment Options in Neurology, 9, 325-336.

Caballero, C.V. (2004). Costos en la artritis reumatoide. En L. Ramírez \& J.M. Anaya (Eds.), Artritis Reumatoide (pp. 521-524). Medellín, Colombia: Editora Médica Colombiana.

Caballero-Uribe, C.V., Venegas, C., Padilla, A., Paternina, P., Peña, R. \& Peñuela, M. (2004).
Depresión, estrés y desesperanza en pacientes con artritis reumatoide del Caribe colombiano. Revista Colombiana de Reumatología, 11(4), 300-305.

Cadena, J., Cadavid, M., Ocampo, M., Vélez, M.C. \& Anaya, J.M. (2002). Depresión y familia en pacientes con artritis reumatoide. Revista Colombiana de Reumatología, 9, 184-191.

Cadena, J., Vinaccia S., Pérez, A., Rico, M., Hinojosa, H. \& Anaya, J.M. (2003). The Impact of Disease Sctivity on the Quality of Life and Mental Health Status in Colombian Patient with Arthritis Rheumatoid. Journal of Clinical Rheumatology, 9, 142-150.

Connelly, M., Keefe, F.J., Affleck, G., Lumley, M.A., Anderson, T. \& Waters, S. (2007). Effects of Day-to-day Affect Regulation on the Pain Experience of Patients with Rheumatoid Arthritis. Pain, 131(1-2), 162-170.

Coward, D.D. (2003). Facilitation of Self-Transcendence in a Breast Cancer Support Group: II. Oncology Nursing Forum, 30(2), 291-300.

Coward, D.D. (2007). Teoría de la autotrascendencia. En A. Marriner \& M. Raile (Eds.), Modelos y teorías en enfermería (pp. 647-667). Madrid, España: Elsevier.

Cutolo, M. \& Straub, R.H. (2006). Stress as a Risk Factor in the Pathogenesis of Rheumatoid Arthritis. Neuroimmunomodulation, 13(5-6), 277-282.

Davis, G.C. (2003). Improved Sleep May Reduce Arthritis Pain. Holistic nursing practice, 17(3), 128-135.

Delgado-Vega, A.M., Martín, J., Granados, J. \& Anaya, J.M. (2006). Epidemiología genética de la artritis reumatoide: ¿Qué esperar de América Latina? Biomédica, 26, 562-584.

Escalante, A. \& Del Rincón, I. (1999). How Much Disability in Rheumatoid Arthritis is Explai- 
ned by Rheumatoid Arthritis. Arthritis \& Rheumatism, 42, 1712-1721.

Fredrickson, B.L. (1998). What Good are Positive Emotions? Review of General Psychology, 2, 300-319.

Fredrickson, B.L. (2001). The Role of Positive Emotions in Positive Psychology: The Broadenand-build Theory of Positive Emotions. American Psychologist, 56, 218-226.

Fredrickson, B.L. (2004). Gratitude, Like Other Positive Emotions, Broadens and Builds. En R.A. Emmons \& M.E. McCullough (Eds.). The Psychology of Gratitude (pp. 145-166). New York: Oxford University Press.

Fredrickson, B.L. \& Losada, M.F. (2005). Positive Affect and the Complex Dynamics of Human Flourishing. American Psychologist, 60(7), 678-686.

Geenen, R., van Middendorp, H. \& Bijlsma, J.W. (2006). The Impact of Stressors on Health Status and Hypothalamic-pituitary-adrenal Axis and Autonomic Nervous System Responsiveness in Rheumatoid Arthritis. Annals of the New York Academy of Sciences, 1069, 7797.

Gómez, L., Pérez, M. \& Vila, J. (2001). Problemática actual del apoyo social y su relación con la salud: una revisión. Psicología conductual, 9(1), 5-38.

González, H. (2004). Factores psicosociales en la artritis reumatoide. En L. Ramírez \& J.M. Anaya (Eds.). Artritis Reumatoide (pp. 301305). Medellín, Colombia: Editora Médica Colombiana.

Groarke, A., Curtis, R., Coughlan, R. \& Gsel, A. (2004). The Role of Perceived and Actual Disease Status in Adjustment to Rheumatoid Arthritis. Rheumatology, 43(9), 1142-1149.

Gutiérrez, L.F. \& Londoño, J.D. (2004). Epidemiología de la artritis reumatoide. En L. Ramírez
\& J.M. Anaya (Eds.). Artritis Reumatoide (pp. 25-29). Medellín, Colombia: Editora Médica Colombiana.

Hill, C.L., Gill, T., Taylor, A.W., Daly, A., Dal Grande, E. \& Adams, R.J. (2007). Psychological Factors and Quality of Life in Arthritis: a Population-based Study. Clinical Rheumatology, 26(7), 1049-1054.

Hommel, K.A., Wagner, J.L., Chaney, J.M., White, M.M. \& Mullins, L.L. (2004). Perceived Importance of Activities of Daily Living and Arthritis Helplessness in Rheumatoid Arthritis; a Prospective Investigation. Journal of Psychosomatic Research, 57(2), 159-164.

Horner, K.L. (1998). Individuality in Vulnerability: Influences on Physical Health. Journal of Health Psychology, 3(1), 71-85.

Isik, A., Koca, S.S., Ozturk, A. \& Mermi, O. (2007). Anxiety and Depression in Patients with Rheumatoid Arthritis. Clinical Rheumatology, 26, 872-878.

Juth, V., Smyth, J.M. \& Santuzzi, A.M. (2008). How Do You Feel? Self-esteem Predicts Affect, Stress, Social Interaction, and Symptom Severity during Daily Life in Patients with Chronic Illness. Journal of Health Psychology, 13(7), 884-894.

Kralik, D., van Loon, A. \& Visentin, K. (2006). Resilience in the Chronic Illness Experience. Educational Action Research, 14(2), 187-201.

Krol, B., Sanderman, R. \& Suurmeijer, T.P. (1993). Social Support, Rheumatoid Arthritis and Quality of Life: Concepts, Measurement and Research. Patient Education and Counseling, 20(2-3), 101-120.

Leight, J., Frics, J. \& Parkh, N. (1992). Severity of Disability in the Rheumatology Arthritis. Journal of Rheumatology, 19, 1906-1911.

Lundman, B., Aléx, L., Jonsén, E., Norberg, A., Nygren, B., Santamaki, R. et ál. (2010). Inner 
Strength: A Theoretical Analysis of Salutogenic Concepts. International Journal of Nursing Studies, 47(2), 251-260.

Maddux, J.E. \& Gosselin, J.T. (2003). Self-efficacy. En M.R. Leary \& J.P. Tangney (Eds.). Handbook of self and identity (pp. 218-238). New York: Guilford Press.

Minnock, P., FitzGerald, O. \& Bresnihan, B. (2003). Quality of Life, Social Support, and Knowledge of Disease in Women with Rheumatoid Arthritis. Arthritis \& Rheumatism, 49(2), 221-227.

Murphy, H., Dickens, C., Creed, F. \& Bernstein, R. (1999). Depression, Illness Perception and Coping in Rheumatoid Arthritis. Journal of Psychosomatic Research, 46(2), 155-164.

Neill, J. (2002). Transcendence and Transformation in the Life Patterns of Women Living with Rheumatoid Arthritis. Journal of Advanced Nursing, 24(4), 27-47.

Neill, J. (2005). Health as Expanding Consciousness: Seven Women Living With Multiple Sclerosis or Rheumatoid Arthritis. Nursing Science Quarterly, 18(4), 334-343.

Pelechano, V. (2008). Enfermedades crónicas y psicología. Madrid, España: Klinik.

Philippe, F.L., Lecours, S. \& Beaulieu-Pelletier, G. (2009). Resilience and Positive Emotions: Examining the Role of Emotional Memories. Journal of Personality, 77(1), 139-176.

Pollard, L.C., Choy, E.H., Gonzalez, J., Khoshaba, B. \& Scott, D.L. (2006). Fatigue in Rheumatoid Arthritis Reflects Pain, not Disease Activity. Rheumatology, 45, 885-889.

Quiceno, J.M. (2010). Diseño y evaluación de un programa de potenciación de la resiliencia para pacientes con diagnóstico de artritis reumatoide (Tesis doctoral inédita). Universidad Autónoma de Madrid, España.
Quiceno, J.M. \& Vinaccia, S. (2010). Percepcion de enfermedad: una aproximación a partir del "Illness Perception Questionnaire". Psicología desde el Caribe, 25, 56-83.

Ramos, F. (1999). Enfermedades Reumáticas. México, D.F.: Mc Graw-Hill.

Reed, P.G. (1987). Constructing a Conceptual Framework for Psychosocial Nursing. Journal of Psychosocial Nursing, 25(2), 24-28.

Reed, P.G. (1991). Toward a Nursing Theory of Self-transcendence: Deductive Reformulation Using Developmental Theories. Advances in Nursing Science, 13(4), 64-77.

Reed, P.G. (2003). The Theory of Self-Transcendence. En M.J. Smith \& P. Liehr. (Eds.). Middle Range Theories in Nursing. New York: Springer.

Rodríguez-Marín, J. \& Neipp, M.C. (2008). Manual de psicología social de la salud. Madrid, España: Editorial Síntesis.

Rojas-Villarraga, A., Díaz, F.J., Calvo-Páramo, E., Salazar, J.C., Iglesias-Gamarra, A., Mantilla, R.D., et ál. (2009). Familial Disease, the HLADRB1 Shared Epitope and Anti-CCP Antibodies Influence Time at Appearance of Substantial Joint Damage in Rheumatoid Arthritis. Journal of Autoimmunity, 32, 64-69.

Saroglou, V., Buxant, C. \& Tilquin, J. (2008). Positive Emotions as Leading to Religion and Spirituality. The Journal of Positive Psychology, 3(3), 165-173.

Scharloo, M., Kaptein, A.A., Weinman, J.A., Hazes, J.M., Breedveld, F.C. \& Rooijmans, H.G.M. (1999). Predicting Functional Status in Patients with Rheumatoid Arthritis. Journal Rheumatology, 26(8), 1686-1693.

Scharloo, M., Kaptein, A.A., Weinman, J., Hazes, J.M., Willems, L.N.A., Bergman, W., et ál. (1998). Illness Perceptions, Coping and 
Functioning in Patients with Rheumatoid Arthritis, Chronic Obstructive Pulmonary Disease and Psoriasis. Journal of Psychosomatic Research, 44(5), 573-585.

Sharpe, L., Sensky, T. \& Allard, S. (2001). The Course of Depression in Recent Onset Rheumatoid Arthritis - The Predictive Role of Disability, Illness Perceptions, Pain and Coping. Journal of Psychosomatic Research, 51, 713719.

Simeon, D., Yehuda, R., Cunill, R., Knutelska, M., Putnam, F.W. \& Smith, L.M. (2007). Factors Associated with Resilience in Healthy Adults. Psychoneuroendocrinology, 32(8-10), 11491152.

Smith, B.W. \& Zautra, A.J. (2002). The Role of Personality in Exposure and Reactivity to Interpersonal Stress in Relation to Arthritis Disease Activity and Negative Affect in Women. Health Psychology, 21(1), 81-88.

Smith, B.W. \& Zautra, A.J. (2008). Vulnerability and Resilience in Women with Arthritis: Test of a Two-Factor Model. Journal of Consulting and Clinical Psychology, 76(5), 799-810.

Steptoe, A., Dockray, S. \& Wardle, J. (2009). Positive Affect and Psychobiological Processes Relevant to Health. Journal of Personality, 77(6), 1747-1476.

Strand, E.B., Zautra, A.J., Thoresen, M., Odegard, S., Uhlig, T. \& Finset, A. (2006). Positive Affect as a Factor of Resilience in the Painnegative Affect Relationship in Patients with Rheumatoid Arthritis. Journal Psychosomatic Research, 60(5), 477-484.

Strating, M.M., Suurmeijer, T.P. \& van Schuur, W.H. (2006). Disability, Social Support, and Distress in Rheumatoid Arthritis: Results from a Thirteen-year Prospective Study. Arthritis \& Rheumatism, 55(5), 736-744.
Straub, R.H. \& Cutolo, M. (2001). Involvement of the Hypothalamic-pituitary-adrenal/gonadal Axis and the Peripheral Nervous System in Rheumatoid Arthritis: Viewpoint Based on a Systemic Pathogenetic Role. Arthritis and Rheumatism, 44(3), 493-507.

Straub, R.H. \& Kalden, J.R. (2009). Stress of Different Types Increases the Proinflammatory Load in Rheumatoid Arthritis. Arthritis Research \& Therapy, 11(3), 114-115.

Taylor, S.E. (2007). Psicología de la Salud (6ª ed.). México, D.F.: McGraw-Hill.

Teixeira, M.E. (2008). Self-Transcendence a Concept Analysis for Nursing Praxis. Holistic Nursing Practice, 22(1), 25-31.

Treharne, G.J., Lyons, A.C., Haleb, E.D., Douglas, K.M., Goodchild, C.E., Booth, D.A. et ál. (2007). Sleep Disruption Frequency in Rheumatoid Arthritis: Perceived Stress Predicts Poor Outcome Over one Year. Musculoskeletal Care, 5(1), 51-64.

Van Middendorp, H., Geenen, R., Sorbi, M.J., van Doornen, L.J.P. \& Bijlsma, J.W.J. (2005). Emotion Regulation Predicts Change of Perceived Health in Patients with Rheumatoid Arthritis. Annals of the Rheumatic Diseases, 64, 10711074.

Vera, B., Carbelo, B. \& Vecina, M.L. (2006). La experiencia traumática desde la psicología positiva: resiliencia y crecimiento postraumático. Papeles del Psicólogo, 27(1), 40-49.

Wolfe, F. \& Michaud, K. (2009). Predicting Depression in Rheumatoid Arthritis: the Signal Importance of Pain Extent and Fatigue, and Comorbidity. Arthritis and Rheumatism, 61(5), 667-673.

Wright, L.J., Zautra, A.J. \& Going, S. (2008). Adaptation to Early Knee Osteoarthritis: The Role of Risk, Resilience, and Disease Severity on Pain and Physical Functioning. Annals of Behavioral Medicine, 36(1), 70-80. 
Zautra, A.J., Fasman, R., Parish, B.P. \& Davis, M.C. (2007). Daily Fatigue in Women with Osteoarthritis, Rheumatoid Arthritis, and Fibromyalgia. Pain, 128, 128-135.
Zyrianova, Y., Kelly, B.D., Gallagher, C., McCarthy, C., Molloy, M.G., Sheehan, J. et ál. (2006). Depression and Anxiety in Rheumatoid Arthritis: the Role of Perceived Social Support. Irish Journal of Medical Science, 175(2), 32-36. 